\title{
Is neoadjuvant androgen deprivation therapy beneficial in prostate cancer treated with definitive radiotherapy?
}

\author{
Keun-Yong Eom, MD1, Sung W Ha, MD ${ }^{1,2,3}$, Eunsik Lee, MD, Cheol Kwak, MD4, Sang Eun Lee, MD \\ 'Department of Radiation Oncology, Seoul National University College of Medicine, Seoul; \\ ${ }^{2}$ Institute of Radiation Medicine, Medical Research Center, Seoul National University, Seoul; \\ ${ }^{3}$ Cancer Research Institute, Seoul National University College of Medicine, Seoul; \\ ${ }^{4}$ Department of Urology, Seoul National University College of Medicine, Seoul; \\ ${ }^{5}$ Department of Urology, Seoul National University Bundang Hospital, Seongam, Korea
}

Purpose: To determine whether neoadjuvant androgen deprivation therapy (NADT) improves clinical outcomes in patients with prostate cancer treated with definitive radiotherapy.

Materials and Methods: We retrospectively reviewed medical records of 201 patients with prostate cancer treated with radiotherapy between January 1991 and December 2008. Of these, 156 patients with more than 3 years of follow-up were the subjects of this study. The median duration of follow-up was 91.2 months. NADT was given in 103 patients (66\%) with median duration of 3.3 months (range, 1.0 to 7.7 months). Radiation dose was escalated gradually from 64 Gy to 81 Gy using intensitymodulated radiotherapy technique.

Results: Biochemical relapse-free survival (BCRFS) and overall survival (OS) of all patients were $72.6 \%$ and $90.7 \%$ at 5 years, respectively. BCRFS and OS of NADT group were $79.5 \%$ and $89.8 \%$ at 5 years and those of radiotherapy alone group were $58.8 \%$ and $92.3 \%$ at 5 years, respectively. Risk group $(p=0.010)$ and radiation dose $\geq 70 \mathrm{~Gy}(p=0.017)$ affected BCRFS independently. NADT was a significant prognostic factor in univariate analysis, but not in multivariate analysis $(p=0.073)$. Radiation dose $\geq 70 \mathrm{~Gy}$ was only an independent factor for OS ( $p=0.007$; hazard ratio, $0.261 ; 95 \%$ confidence interval, $0.071-0.963$ ).

Conclusion: NADT prior to definitive radiotherapy did not result in significant benefit in terms of BCRFS and OS. NADT should not be performed routinely in the era of dose-escalated radiotherapy.

Keywords: Prostate cancer, Radiotherapy, Neoadjuvant androgen deprivation, Radiation dose

\section{Introduction}

Androgen deprivation therapy (ADT) after definitive radiotherapy (RT) has been proven to be beneficial in patients with high-risk prostate cancer [1-5] and offering 2-3 years of adjuvant ADT after definitive RT is a standard treatment in these patients. In addition, ADT prior to definitive local treatment prolongs disease-free survival in high-risk prostate cancer $[6,7]$. Successful application of neoadjuvant androgen deprivation therapy (NADT) to high-risk prostate cancer promoted the use of NADT in low- to intermediate-risk prostate cancer. Although growing number of patients with intermediate- to high-risk prostate cancer undergoes NADT [8], the role of NADT in these patients has been undefined.

Recently, some phase III randomized studies showed positive prognostic effects of NADT in these patients [9-11]

Received 16 March 2014, Revised 31 May 2014, Accepted 9 December 2014.

Correspondence: Sung W Ha, MD, PhD, Department of Radiation Oncology, Seoul National University College of Medicine, 103 Daehak-ro, Jongno-gu, Seoul 110-799, Korea. Tel: +82-2-2072-2524, Fax: +82-2-765-3317, E-mail: swha@snu.ac.kr

(c) This is an Open Access article distributed under the terms of the Creative Commons Attribution Non-Commercial License (http://creativecommons.org/ licenses/by-nc/3.0/) which permits unrestricted non-commercial use, distribution, and reproduction in any medium, provided the original work is properly cited.

www.e-roj.org 
However, the question of what will be the optimal in low- to intermediate-risk and even in high-risk prostate cancer still remains because RT technique has evolved for decades to allow delivering higher radiation dose to the prostate, which improves treatment efficacy without severe gastrointestinal (GI) or genitourinary (GU) toxicities. Conventional radiation dose ranging from 60 Gy to 70 Gy which was adopted in most randomized studies comparing NADT plus RT vs. RT alone, is now regarded as suboptimal in the treatment of prostate cancer $[12,13]$. Nowadays, standard radiation dose reaches at least 74 Gy or higher in patients with prostate cancer [1214] as several randomized studies comparing conventional vs. dose-escalated RT have confirmed the benefit of the latter [1517]. Therefore, we performed retrospective analysis to evaluate the effect of NADT according to risk groups and investigated whether NADT improves clinical outcomes of prostate cancer in patients who underwent definitive RT with or without adjuvant ADT.

\section{Materials and Methods}

We retrospectively reviewed medical records of newly diagnosed patients with adenocarcinoma of the prostate treated at the Department of Radiation Oncology between January 1991 and December 2008. Two hundred and one patients were identified on the local database. Of these, 156 patients, who had CT1-T4, N0, and Mo by the American Joint Committee on Cancer seventh edition staging system [18] with more than 3 years of follow-up except for 4 patient $s$ who had died of disease, were the subjects of this study. The median follow-up was 91.2 months (range, 14.4 to 180.2 months) and the mean age of all patients was 69.4 years (range, 48.7 to 87.6 years).

Table 1. Demographics of all the patients and clinicopathologic features

\begin{tabular}{|c|c|c|c|c|}
\hline Characteristic & Total $(n=156)$ & NADT (-) $(n=53)$ & NADT $(+)(n=103)$ & $p$-value \\
\hline Age $(y r)$ & $69.4(48.7-87.6)$ & $68.7(48.7-82.6)$ & $69.8(50.8-87.6)$ & 0.380 \\
\hline ECOG PS & & & & 0.011 \\
\hline 0 & $42(26.9)$ & $8(15.1)$ & $34(33.0)$ & \\
\hline 1 & $112(71.8)$ & $43(81.1)$ & $69(67.0)$ & \\
\hline 2 & $2(1.3)$ & $2(3.8)$ & $0(0)$ & \\
\hline T Stage $e^{a)}$ & & & & 0.066 \\
\hline $\mathrm{T} 1 \mathrm{~b}$ & $1(0.6)$ & $1(1.9)$ & $0(0.0)$ & \\
\hline T1c & $37(23.7)$ & $15(28.3)$ & $22(21.4)$ & \\
\hline T2a & $29(18.6)$ & $14(26.4)$ & $15(14.6)$ & \\
\hline $\mathrm{T} 2 \mathrm{~b}$ & $18(11.5)$ & $6(11.3)$ & $12(11.7)$ & \\
\hline $\mathrm{T} 2 \mathrm{c}$ & $26(16.7)$ & $6(11.3)$ & $20(19.4)$ & \\
\hline ТЗа & $12(7.7)$ & $6(11.3)$ & $6(5.8)$ & \\
\hline T3b & $27(17.3)$ & $4(7.5)$ & $23(22.3)$ & \\
\hline T4 & $6(3.8)$ & $1(1.9)$ & $5(4.9)$ & \\
\hline Gleason score & & & & 0.013 \\
\hline $2-6$ & $58(37.2)$ & $28(52.8)$ & $30(29.1)$ & \\
\hline 7 & $46(29.5)$ & $13(24.5)$ & $33(32.0)$ & \\
\hline $8-10$ & $52(33.3)$ & $12(22.6)$ & $40(38.8)$ & \\
\hline Pretreatment PSA (ng/mL) & & & & 0.696 \\
\hline$\leq 10$ & $59(37.8)$ & $21(39.6)$ & $38(36.9)$ & \\
\hline $10-20$ & $43(27.6)$ & $16(30.2)$ & $27(26.2)$ & \\
\hline$>20$ & $54(34.6)$ & $16(30.2)$ & $38(36.9)$ & \\
\hline Risk group ${ }^{b)}$ & & & & 0.116 \\
\hline Low & $18(11.5)$ & $9(17.0)$ & $9(8.7)$ & \\
\hline Intermediate & $42(26.9)$ & $17(32.1)$ & $25(24.3)$ & \\
\hline High & $96(61.5)$ & $27(50.9)$ & $69(67.0)$ & \\
\hline Radiation dose (Gy) & $68(64-81)$ & $67(64-81)$ & $70(64-81)$ & 0.000 \\
\hline
\end{tabular}

Values are presented as median (range) or number of patients (\%).

NADT, neoadjuvant androgen deprivation therapy; ECOG PS, Eastern Cooperative Oncology Group performance status; PSA, prostate specific antigen.

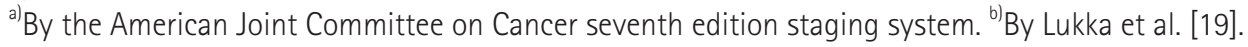


The characteristics of all patients are summarized in Table 1.

Pretreatment evaluation included prostate needle biopsy or transurethral resection, bone scan, chest radiograph, pelvic computed tomography (CT) scan and/or pelvic magnetic resonance image (MRI) scan, and pretreatment prostate specific antigen (PSA). The risk groups [19] were defined according to combinations of each pretreatment variables including $T$ stage, Gleason score, and pretreatment PSA as follows: low-risk was Gleason score $\leq 6$ and PSA $\leq 10 \mathrm{ng} / \mathrm{mL}$ and stage T1-T2a; Intermediate-risk was Gleason score $\leq 7$ and/or PSA between 10 and $20 \mathrm{ng} / \mathrm{mL}$ and/or stage T1-T2, not low-risk; high-risk was Gleason score $\geq 8$ or PSA $>20 \mathrm{ng} / \mathrm{mL}$ or stage $\geq \mathrm{T} 3 \mathrm{a}$.

\section{Neoadjuvant, concurrent, and adjuvant ADT}

NADT was given in 103 patients (66.0\%) prior to RT. Complete androgen blockade using gonadotropin-releasing hormone agonist combined with anti-androgen agent was performed in most patients $(n=84)$. Eighteen patients underwent antiandrogen agent alone. Surgical NADT (bilateral orchiectomy) was performed in 1 patient. The median duration of NADT was 3.3 months (range, 1.0 to 7.7 months). Concurrent ADT during RT following NADT was performed in 74 patients (71.8\%). Adjuvant ADT was given in 59 patients (57.3\%) with median duration of 6.3 months (range, 1.0 to 61.9 months). No patient received adjuvant ADT alone after definitive RT. Patients with good performance status, higher T stage and/or higher Gleason score were more susceptible to NADT. However, in other risk factors including age, pretreatment PSA and risk group, there was no statistical difference between two groups.

\section{Definitive RT}

RT was performed with 1.8 Gy per fraction in 7-10 weeks with or without pelvic irradiation using megavoltage machines. Since three-dimensional conformal radiation therapy (3D-CRT) and/or intensity-modulated radiation therapy (IMRT) had become available using planning CT scans and radiation treatment planning system, the prescription dose to the prostate was escalated step by step. Elective pelvic node irradiation (EPNI) was omitted when the prescription dose exceeds 70 Gy. Until July 2002, the prescription dose to the prostate was less than 70 Gy and EPNI was performed in all patients. Four-field box technique using 6-10 MV photon beams was applied to the pelvis with the median dose of 45.0 Gy (range, 30.6 to $50.4 \mathrm{~Gy}$ ). At the completion of EPNI, multiple portals or arc technique was utilized to treat the prostate only with the median dose of $67.0 \mathrm{~Gy}$ (range, 64.0 to $68.4 \mathrm{~Gy}$ ).
Between August 2002 and February 2004, the prescription dose was escalated to 70.2 Gy without EPNI using six coplanar radiation portals. The seminal vesicles were treated with the median dose of $45.0 \mathrm{~Gy}$ (range, 0 to $59.4 \mathrm{~Gy}$ ). After March 2004 , the prescription dose was escalated to 75.6 or $81.0 \mathrm{~Gy}$ without EPNI using IMRT technique with 6 MV photons. Thirtyeight patients were treated using IMRT technique. The median dose to seminal vesicle (SV) was 50.4 Gy (range, 45.0 to 50.4 Gy). In IMRT planning, no more than $30 \%$ of rectal wall and $50 \%$ of bladder wall received $95 \%$ of prescribed dose.

After definitive RT, patients were scheduled to visit outpatient clinic at regular intervals, which was 4 months at 1-2 years, 6 months at 3-5 years, and one year thereafter. Treatment toxicities during and after RT were assessed by Common Terminology Criteria of Adverse Event v3.0. Treatment toxicities developed within 90 days after completion of RT were regarded as acute whereas the others did as late.

\section{Statistical analyses}

The statistical analysis was performed using the SPSS software ver. 18.0.0 (SPSS Inc. Chicago, IL, USA). A p-value less than 0.05 was considered statistically significant. Categorical and continuous data were compared between the groups using Fisher exact test and Student t-test, respectively. The survival curves were generated using the Kaplan-Meier method and compared with log-rank test. The Cox proportional hazards model was adopted for multivariate analysis. To adjust for confounding factors between two groups, we incorporated a propensity score in the Cox proportional hazards model, which estimates the tendency to perform NADT. The propensity score was the linear predictor from a multivariate binary logistic model in which the response variable was NADT and the covariates were patient's age, the Eastern Cooperative Oncology Group performance status, clinical T stage, Gleason score, and pretreatment PSA.

\section{Results}

\section{Biochemical relapse, clinical failures and overall survival} Biochemical relapse (BCR) was defined as the Phoenix definition of $\geq$ PSA nadir $+2 \mathrm{ng} / \mathrm{mL}$. Biochemical relapsefree survival (BCRFS) and overall survival (OS) of all patients were $72.6 \%$ and $90.7 \%$ at 5 years, respectively (Fig. 1). BCRFS of patients was 92.9\%, 79.5\%, and 66.7\% at 5 years for low-, intermediate-, and high-risk, respectively (Fig. 2).

In low-risk group of 18 patients, only one patient who underwent NADT followed by definitive RT with 75.6 Gy had 


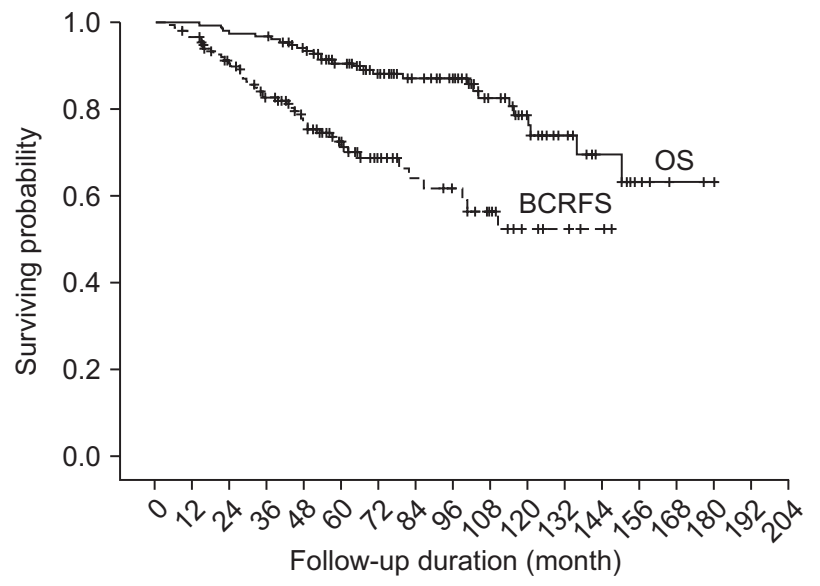

Fig. 1. The graph shows biochemical relapse-free survival (BCRFS) and overall survival (OS) of all patients generated by Kaplan-Meier methods. At 5 years, BCRFS and OS were $72.6 \%$ and 90.7\%. RT, radiotherapy; NADT, neoadjuvant androgen deprivation therapy.

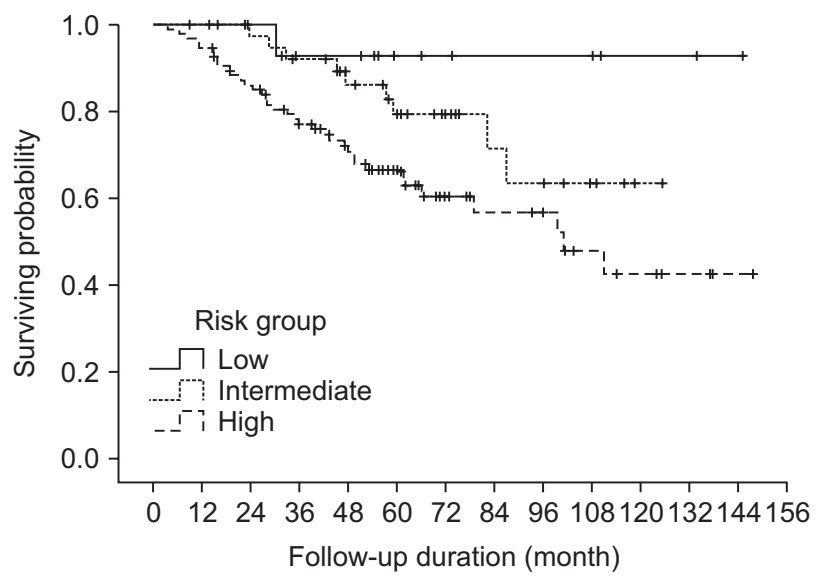

Fig. 2. The graph shows biochemical relapse-free survival (BCRFS) according to the risk groups. The BCRFS was 92.9\%, 79.5\%, and $66.7 \%$ at 5 years for low-, intermediate-, and high-risk groups, respectively.

Table 2. Patterns of biochemical relapse in regard to NADT, radiation dose, and risk group

\begin{tabular}{|c|c|c|c|c|c|c|}
\hline \multirow{2}{*}{ Risk group ${ }^{a)}$} & \multicolumn{3}{|c|}{$<70$ Gy $(n=79)$} & \multicolumn{3}{|c|}{$\geq 70$ Gy $(n=77)$} \\
\hline & NADT (-) & NADT (+) & $p$-value $e^{b)}$ & NADT (-) & NADT (+) & $p$-value \\
\hline Low & 0/4 (0.0) & 0/2 (0.0) & 1.000 & $0 / 5(0.0)$ & 1/7 (14.3) & 1.000 \\
\hline Intermediate & 8/15 (53.3) & $1 / 11(9.1)$ & 0.0362 & 0/2 (0.0) & 0/14 (0.0) & 1.000 \\
\hline High & $17 / 23(73.9)$ & $11 / 24(45.8)$ & 0.0753 & $1 / 4(25.0)$ & $7 / 45$ (15.6) & 0.5220 \\
\hline
\end{tabular}

Values are presented as number of patients (\%).

NADT, neoadjuvant androgen deprivation therapy.

a) By Lukka et al. [19]. ${ }^{\text {b) }}$ Fisher exact test.

developed BCR at 30 months of follow-up. He was salvaged with hormonal therapy and achieved PSA nadir less than 1.0 $\mathrm{ng} / \mathrm{mL}$ again. Local (LF) or regional failure was not observed in low-risk group. Distant failure (DF) had developed at 48 months after treatment in one patient, who was salvaged with wedge resection of pulmonary metastasis.

Among 42 patients with intermediate-risk features, 9 (21.4\%) BCR, 1 (2.4\%) LF, and 2 (4.8\%) DF were observed. In patients treated with less than $70 \mathrm{~Gy}, 53.3 \%$ (8 of 15) of RT alone group had BCR whereas $9.1 \%$ (1 of 11) of NADT followed by RT group had BCR with statistically significant difference $(p=0.036)$. Only one patient had adjuvant ADT longer than 1 year. The BCRFS of patients treated with radiation dose less than $70 \mathrm{~Gy}$ with and without NADT was $95.0 \%$ and $57.4 \%$ at 5 years, respectively $(p=0.004)$. Sites of DF were lungs in one patient and bone in the other. All clinical failures were preceded by BCR and all patients with BCR underwent salvage hormonal therapy. After hormonal therapy, PSA nadir of the 5 patients had reached below $1.0 \mathrm{ng} / \mathrm{mL}$.
In 96 patients of high-risk group, BCR rate was around $40 \%$. Table 2 shows BCR in regard of NADT, radiation dose, and risk groups. In patients treated with less than $70 \mathrm{~Gy}, 73.9 \%$ (17 of 23) of RT alone group had BCR whereas 45.8\% (11 of 24) of NADT followed by RT group had BCR with marginal statistical difference $(p=0.075)$. The BCRFS of patients treated with radiation dose less than $70 \mathrm{~Gy}$ with and without NADT was $72.9 \%$ and $50.4 \%$ at 5 years, respectively $(p=0.003)$. Among 4 patients receiving radiation dose $\geq 70$ Gy without NADT, one patient (25.0\%) had BCRFS while 7 of 45 patients (15.6\%) receiving radiation dose $\geq 70$ Gy with NADT had BCRFS. The difference of BCRFS between these groups was not statistically significant in univariate analysis ( $p=0.166)$.

The effect of total duration of ADT was evaluated in highrisk patients. There were 70 patients $(72.9 \%)$ receiving shorter than 1 year of ADT and 26 patients receiving longer than 1 year of ADT. There was no difference of BCRFS between the two groups ( $67.4 \%$ vs. $64.6 \%$ at 5 years, $p=0.980$ ). BCRFS of 85 patients (88.5\%) with longer than 2 years of ADT was 
90.0\% which is higher than $63.8 \%$ of 11 patients with shorter than 2 years of ADT $(p=0.111)$. However, when stratified by radiation dose, there was no difference as well.

As to clinical failures, $11(11.5 \%)$ LF, 8 (7.8\%) DF, and 3 (3.1\%) with LF and DF were observed. All clinical failures were preceded by BCR. Most of the patients with BCR underwent life-long salvage hormonal therapy. Among 37 patients with $\mathrm{BCR}, 7$ patients did not undergo salvage hormonal therapy because of patient's refusal in 6 patients and heart problem in one. One patient with local failure confirmed at needle biopsy had been salvaged by retropubic prostatectomy.

\section{Effect of NADT and prognostic factors for BCRFS and OS} BCRFS and OS of NADT group were $79.5 \%$ and $89.8 \%$ at 5 years. Those of RT alone group were $58.8 \%$ and $92.3 \%$ at 5 years, respectively.

In univariate analysis for BCRFS, patients' age, risk group, NADT, and radiation dose were statistically significant factors (Table 3). Performance status showed a borderline significance ( $p=0.06)$. Independent prognostic factors affecting BCRFS were risk group and radiation dose in multivariate analysis adjusting propensity score for NADT ( $p=0.010$ and $p=0.017$ respectively) (Table 3 ). NADT trended toward better BCRFS, but did not show statistical significance $(p=0.073)$. When

Table 3. Clinical factors affecting biochemical relapse-free survival in all patients

\begin{tabular}{|c|c|c|c|}
\hline & \multirow{2}{*}{$\begin{array}{c}\text { Univariate } \\
\mathrm{p} \text {-value }\end{array}$} & \multicolumn{2}{|c|}{ Multivariate } \\
\hline & & $p$-value & Hazard ratio $(95 \% \mathrm{Cl})$ \\
\hline Age & 0.001 & 0.995 & \\
\hline ECOG PS & 0.060 & 0.152 & \\
\hline Stage & 0.461 & - & \\
\hline Gleason score & 0.120 & - & \\
\hline Pretreatment PSA & 0.131 & - & \\
\hline Risk group ${ }^{a)}$ & 0.017 & 0.010 & \\
\hline Intermediate & & 0.089 & 7.078 (0.740-67.697) \\
\hline High & & 0.007 & 51.439 (2.913-908.450) \\
\hline NADT & 0.001 & 0.073 & $0.532(0.267-1.062)$ \\
\hline ADT & 0.122 & 0.407 & \\
\hline Radiation dose $\geq 70$ Gy & 0.000 & 0.017 & $0.361(0.157-0.830)$ \\
\hline
\end{tabular}

ECOG PS, Eastern Cooperative Oncology Group performance status; PSA, prostate-specific antigen; NADT, neoadjuvant androgen deprivation therapy; $A D T$, adjuvant androgen deprivation; $\mathrm{Cl}$, confidence interval.

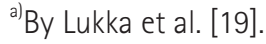

A

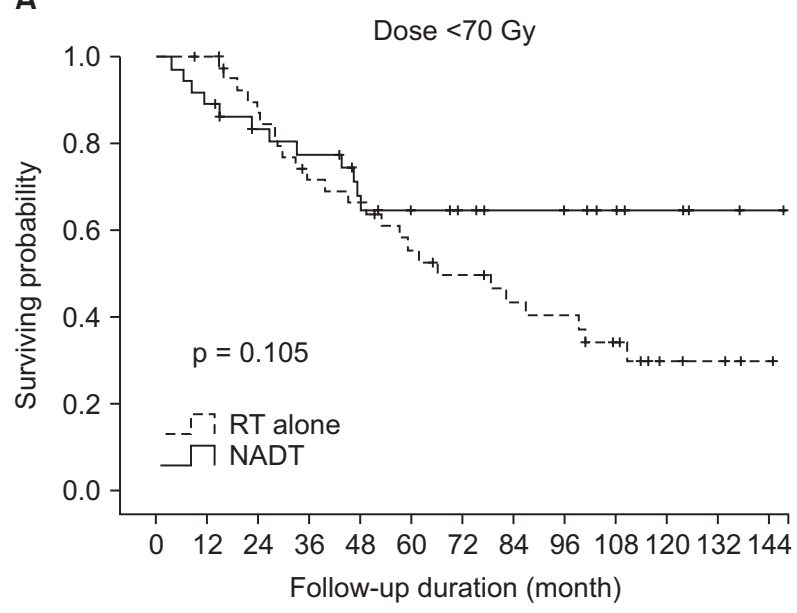

B

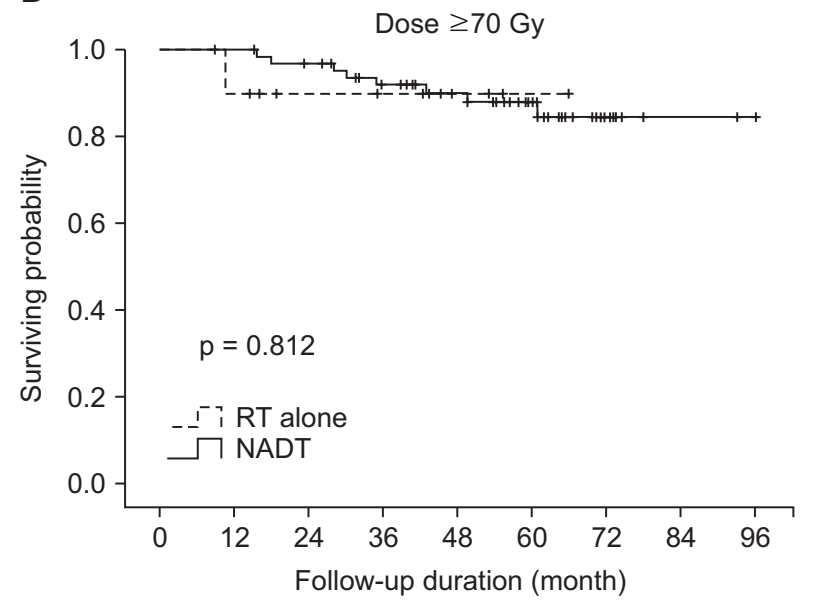

Fig. 3. The graphs show biochemical relapse-free survival according to the use of neoadjuvant andgrogen deprivation therapy (NADT) stratified by radiation dose, $(A)$ radiation dose $<70$ Gy and $(B)$ radiation dose $\geq 70$ Gy. There was no statistically significant difference between NADT and radiotherapy alone groups. 
stratified by radiation dose, BCRFS of NADT group and RT alone group with radiation dose $<70$ Gy were $64.7 \%$ ( $n=$ $42)$ and $55.5 \%(n=37)$ at 5 years. Those for radiation dose $\geq 70$ Gy were $88.1 \%(n=11)$ and $90.0 \%(n=66)$ at 5 years, respectively. Fig. 3 shows BCRFS curves according to NADT stratified by radiation dose. There was no significant difference between groups with or without NADT in patients receiving radiation dose $\geq 70$ Gy $(p=0.812)$.

OS for NADT and RT alone group were $89.8 \%$ and $92.3 \%$ at 5 years. NADT was not a significant prognostic factor for OS $(p=0.760)$. As for $0 S$, radiation dose was the only significant factor both in univariate and multivariate analysis ( $p=$ 0.007 and $p=0.044$, respectively) (Table 4). Fig. 4 shows $0 S$ difference according to the radiation dose.

\section{Radiation toxicities}

$\mathrm{Gl}$ and $\mathrm{GU}$ toxicities were the majority of the adverse events (Table 5). In patients treated with radiation dose $<70$ Gy and EPNI, 61 (77.2\%) experienced grade 1 or 2 acute GI toxicities, whereas $25(32.5 \%)$ of the others did $(p=0.000)$. Of note patients with radiation dose $\geq 70$ Gy had less severe acute $\mathrm{Gl}$ toxicities, which suggests that two possible explanations: EPNI affects negatively on the development of acute GI toxicities and robust dose constraint to organs at risk using IMRT may have preventive effect on the development of acute $\mathrm{Gl}$ toxicities despite higher prescription dose.

Late GI toxicities of grade 1 or 2 were observed in 20 (25.4\%) patients with EPNI and 7 (9.1\%) patients without EPNI ( $p=$ 0.007). Similar to acute $\mathrm{Gl}$ toxicities, late $\mathrm{Gl}$ toxicities were less

Table 4. Clinical factors affecting overall survival in all patients

\begin{tabular}{|c|c|c|c|}
\hline & \multirow{2}{*}{$\begin{array}{c}\text { Univariate } \\
\text { p-value }\end{array}$} & \multicolumn{2}{|c|}{ Multivariate } \\
\hline & & $p$-value & Hazard ratio $(95 \% \mathrm{Cl})$ \\
\hline Age & 0.729 & - & \\
\hline ECOG PS & 0.080 & 0.325 & \\
\hline Stage & 0.515 & - & \\
\hline Gleason score & 0.018 & 0.072 & \\
\hline Pretreatment PSA & 0.222 & - & \\
\hline Risk group ${ }^{a)}$ & 0.192 & - & \\
\hline NADT & 0.760 & 0.843 & \\
\hline ADT & 0.849 & - & \\
\hline Radiation dose $\geq 70 \mathrm{~Gy}$ & 0.007 & 0.044 & $0.261(0.071-0.963)$ \\
\hline
\end{tabular}

ECOG PS, Eastern Cooperative Oncology Group performance status; PSA, prostate-specific antigen; NADT, neoadjuvant androgen deprivation therapy; $A D T$, adjuvant androgen deprivation; $\mathrm{Cl}$, confidence interval.

${ }^{a}$ By Lukka et al. [19].
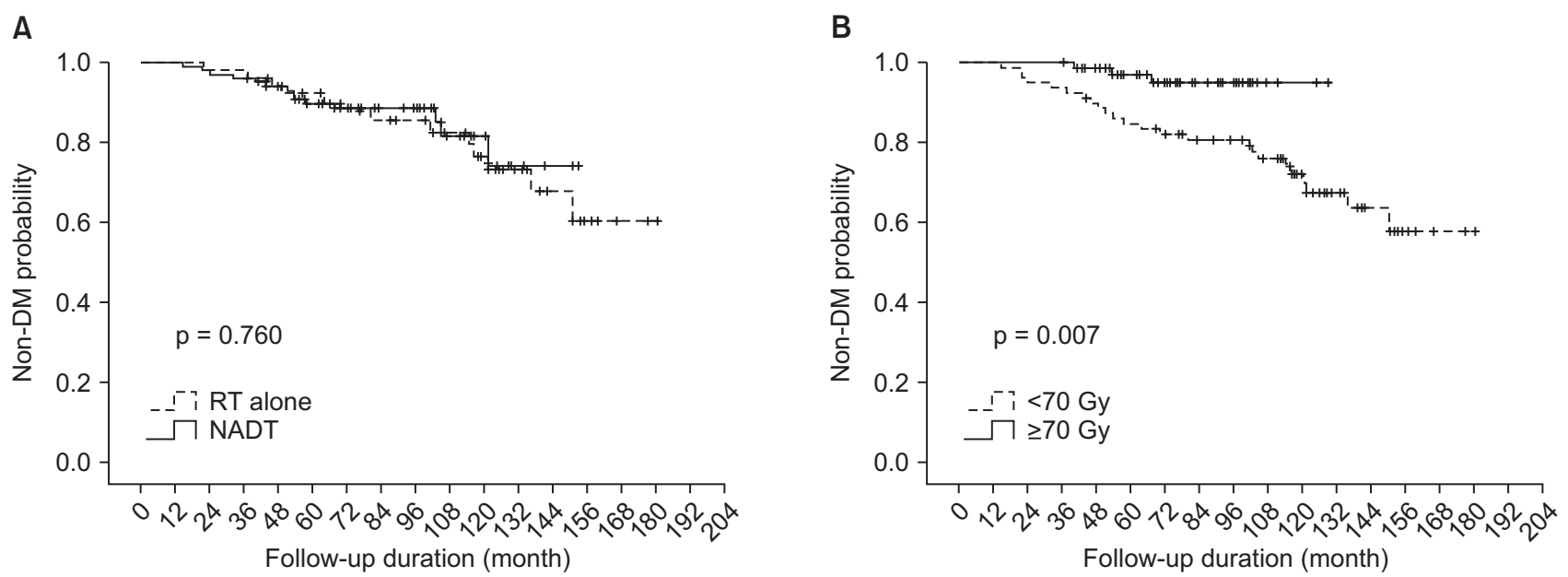

Fig. 4. The graphs show overall survival curves according to (A) the use of neoadjuvant androgen deprivation therapy (NADT) and (B) radiation dose. There was a statistically significant difference between radiation dose $<70$ Gy and radiation dose $\geq 70 \mathrm{~Gy}(p=0.007)$. DM, diabetes mellitus. 
Table 5. Radiation toxicities by Common Toxicity Criteria for Adverse Events (CTCAE) v3.0

\begin{tabular}{|c|c|c|c|c|c|}
\hline \multirow{2}{*}{ Complication } & \multirow{2}{*}{$\begin{array}{l}\text { CTCAE } \\
\text { grade }\end{array}$} & \multicolumn{2}{|c|}{ Radiation dose group } & \multirow{2}{*}{ Total $(n=156)$} & \multirow{2}{*}{ p-value } \\
\hline & & $<70 \mathrm{~Gy}^{\mathrm{a})}(\mathrm{n}=77)$ & $\geq 70$ Gy $(n=77)$ & & \\
\hline \multicolumn{6}{|l|}{ Acute side effects } \\
\hline \multirow[t]{3}{*}{ Gastrointestinal } & 0 & $18(25.7)$ & $52(67.5)$ & $70(44.9)$ & 0.000 \\
\hline & 1 & $54(71.1)$ & $22(28.6)$ & $76(48.7)$ & \\
\hline & 2 & 7 (8.9) & $3(3.9)$ & $10(6.4)$ & \\
\hline \multirow[t]{4}{*}{ Genitourinary } & 0 & $24(30.4)$ & $35(45.5)$ & $59(37.8)$ & 0.196 \\
\hline & 1 & $38(48.1)$ & $30(39.0)$ & $68(43.6)$ & \\
\hline & 2 & $16(20.3)$ & $10(13.0)$ & $26(16.7)$ & \\
\hline & 3 & $1(1.3)$ & $2(2.6)$ & $3(1.9)$ & \\
\hline \multicolumn{6}{|l|}{ Late side effects } \\
\hline \multirow[t]{3}{*}{ Gastrointestinal } & 0 & $59(74.7)$ & $70(90.9)$ & $129(82.7)$ & 0.007 \\
\hline & 1 & $16(20.3)$ & $3(3.9)$ & $19(12.2)$ & \\
\hline & 2 & $4(5.1)$ & $4(5.2)$ & $8(5.1)$ & \\
\hline \multirow[t]{4}{*}{ Genitourinary } & 0 & $56(70.9)$ & $61(79.2)$ & 117 (75.0) & 0.301 \\
\hline & 1 & $10(12.7)$ & $10(13.0)$ & $20(12.8)$ & \\
\hline & 2 & $11(13.9)$ & $6(7.8)$ & $17(10.9)$ & \\
\hline & 3 & $2(2.5)$ & $0(9.0)$ & $2(1.3)$ & \\
\hline
\end{tabular}

Values are presented as number of patients (\%).

${ }^{\text {a) }}$ Patients in this group had received elective pelvic node irradiation.

common in patients with $\geq 70$ Gy compared to patients $<70$ Gy with EPNI ( $p=0.007)$.

Early toxicities of GU system were observed in $62.2 \%$ of all patients. Late GU toxicities had developed in 19 patients $(12.2 \%)$ with grade $2-3$. GU toxicity between dose groups was not different. Treating patients using IMRT technique produced feasible early GU toxicities. Among 38 patients treated with IMRT technique, early GU toxicities of grade 2 to 3 were observed in 7 patients (15.8\%). Toxicities of other organs related to RT were uncommon: early toxicities of grade 1 in 4 patients (2.6\%) and late toxicities of grade 1 in 1 (0.6\%).

\section{Discussion and Conclusion}

The benefit of NADT in high-risk prostate cancer has been confirmed by several randomized studies $[1,7]$, but in low- to intermediate-risk prostate cancer, it is not clearly established. Laverdiere et al. [10] showed in their study, which randomized 120 patients with prostate cancer into three treatment arms (RT vs. NADT plus RT vs. NADT followed by RT and adjuvant $A D T$ ), that NADT was beneficial in terms of response rate to treatment (positive biopsy rate at 12 and 24 months) and median serum PSA level. In another randomized study which enrolled 206 patients with intermediate- to high-risk prostate cancer and implemented 70 Gy of 3D-CRT, D'Amico et al. [9] showed that 6 months of ADT (2 months of neoadjuvant, concomitant, and adjuvant) with RT, when compared to RT alone, achieved an improvement in OS, cause-specific survival (CSS), and survival without salvage ADT. Post-randomization analysis by Nguyen et al. [20] suggested that ADT plus RT was associated with risk reduction from death in intermediate- to high-risk groups. The RTOG 94-08 [11] compared the efficacy of 4 months of short-term ADT before and during RT in 1,979 patients with early prostate cancer. When short-term ADT was given in conjunction with 66.6 Gy of RT, OS, CSS, BCRFS, and distant metastasis-free survival of combination therapy were superior to those of RT alone. From the above randomized studies, there appears that NADT followed by conventional RT improves BCRFS, CSS and OS in patients with intermediate- to high-risk prostate cancer. In this study, NADT showed a trend toward better BCRFS in patients receiving $<70 \mathrm{~Gy}$, which is consistent with the randomized trials.

The clinical benefit of NADT in the era of dose-escalated RT has been unanswered as direct evidences from randomized trials are unavailable. Preliminary results from the French trail GETUG 14 [21], which enrolled 377 patients with intermediaterisk, adopted 80 Gy of dose-escalated RT and investigated the effect of 4 months of NADT, reported improvement of BCRFS (91\% vs. 97\%, $p=0.04$ ) with NADT at 37 months of median follow-up. However, the primary end point of the study, biochemical or clinical control probability, were not statistically significant $(p=0.09)$. Retrospective study of dose- 
escalated RT from Memorial Sloan Kettering Cancer Center [22] suggested that at 99 months of median follow-up, 3 months of NADT had no effect on BCRFS in patients receiving 81 Gy of RT using 5-field IMRT technique. In this study, similar to the data of Memorial Sloan Kettering Cancer Center, the effect of NADT in patients receiving dose-escalated RT did not bring statistically significant improvement of BCRFS, as shown in Fig. 3. Although there was small number of patients in RT alone group in dose-escalated RT group, BCRFS of each group at 5 years was almost same. As of now, the benefit of NADT in dose-escalated RT era is dubious and further studies are warranted.

If NADT is of benefit, how long the duration of NADT will be optimal is more questionable. Randomized studies showed inconsistent results between them. The Irish trial [23] comparing 4 months vs. 8 months of NADT before RT in intermediate- to high-risk prostate cancer, had resulted in no difference in all endpoints including OS, CSS, and BCRFS. Canadian multi-institutional study compared 3 months vs. 8 months of ADT prior to RT in patients with low-to high-risk prostate cancer. Crook et al. [24] demonstrated that pattern of failure did not differ from 3 months to 8 months of NADT. However, the actuarial disease-free survival of 8 months of NADT was higher than that of 3 months of NADT in patients with high-risk features. Interesting finding was observed later in the subsequent analysis of this study. Alexander et al. [25] suggested that biochemical response to NADT before RT, not duration, is critical determinant of BCRFS. This suggestion may explain why randomized studies showed discrepant results between them. If this is the case, risk-adapted NADT might be promising in the future for the treatment of prostate cancer. [26]

Refinement of RT technique prompted dose-escalated RT for the prostate cancer without increasing radiation-related toxicities. Several series [15-17,27] examining dose-escalated RT for the prostate cancer have demonstrated that higher radiation dose results in superior BCRFS to conventional radiation doses. Similar outcomes were seen in this study. RT using conventional dose (<70 Gy) had resulted in unacceptably poor BCRFS, regardless of NADT. Higher radiation dose $(\geq 70$ Gy) had increased BCRFS with hazard ratio of 0.361 ( $p=0.017$; 95\% confidence interval, 0.157-0.830) and increased OS with hazard ratio of 0.261 ( $p=0.062 ; 95 \%$ confidence interval, 0.071-0.963). Patients receiving RT using IMRT technique in this study also showed acceptable GU and GI toxicities.

There are some limitations in this retrospective study. The distribution of risk factors including performance status, clinical T stage and Gleason score was not balanced between patients with and without NADT. Although propensity score was included in the Cox proportional hazard model to adjust these biases, there may still exist some biases that may affect the outcomes of this study. Furthermore, the number of patients in NADT with dose-escalated RT was small that the effect of NADT in these patients could not be fully evaluated. Lastly, toxicities from NADT were not described in this study because unlike the radiation-related toxicities, clinical information regarding toxicities from NADT was insufficient to analyze.

In conclusion, NADT prior to RT trended an improvement of BCRFS in patients receiving radiation dose $<70 \mathrm{~Gy}$ without statistical significance. In patients receiving radiation dose $\geq 70$ Gy, it was of no benefit in BCRFS or OS. The benefit of NADT was questionable even in high-risk patients. On the contrary, dose-escalated RT has resulted in the improvement of BCRFS and OS. In this regard, NADT should not be performed routinely in an era of dose-escalated RT and further investigation is needed to clearly define whether NADT combined with doseescalated RT is beneficial in patients with intermediate- or high-risk prostate cancer.

\section{Conflict of Interest}

No potential conflict of interest relevant to this article was reported.

\section{References}

1. Lawton $C A$, Winter $K$, Murray $K$, et al. Updated results of the phase III Radiation Therapy Oncology Group (RTOG) trial 8531 evaluating the potential benefit of androgen suppression following standard radiation therapy for unfavorable prognosis carcinoma of the prostate. Int J Radiat Oncol Biol Phys 2001:49:937-46.

2. Bolla $M$, de Reijke TM, Van Tienhoven $G$, et al. Duration of androgen suppression in the treatment of prostate cancer. N Engl J Med 2009;360:2516-27.

3. Horwitz EM, Bae K, Hanks GE, et al. Ten-year follow-up of radiation therapy oncology group protocol 92-02: a phase III trial of the duration of elective androgen deprivation in locally advanced prostate cancer. J Clin Oncol 2008;26:2497-504.

4. Bria E, Cuppone F, Giannarelli $D$, et al. Does hormone treatment added to radiotherapy improve outcome in locally advanced prostate cancer? Meta-analysis of randomized trials. Cancer 2009;115:3446-56.

5. Roach M, Lu J, Pilepich MV, et al. Predicting long-term 
survival, and the need for hormonal therapy: a meta-analysis of RTOG prostate cancer trials. Int J Radiat Oncol Biol Phys 2000;47:617-27.

6. Lawton CA, DeSilvio $M$, Roach $M$, et al. An update of the phase III trial comparing whole pelvic to prostate only radiotherapy and neoadjuvant to adjuvant total androgen suppression: updated analysis of RTOG 94-13, with emphasis on unexpected hormone/radiation interactions. Int J Radiat Oncol Biol Phys 2007;69:646-55.

7. Denham JW, Steigler A, Lamb DS, et al. Short-term neoadjuvant androgen deprivation and radiotherapy for locally advanced prostate cancer: 10-year data from the TROG 96.01 randomised trial. Lancet Oncol 2011;12:451-9.

8. Cooperberg MR, Grossfeld GD, Lubeck DP, Carroll PR. National practice patterns and time trends in androgen ablation for localized prostate cancer. J Natl Cancer Inst 2003;95:981-9.

9. D'Amico AV, Manola J, Loffredo M, Renshaw AA, DellaCroce A, Kantoff PW. 6-month androgen suppression plus radiation therapy vs radiation therapy alone for patients with clinically localized prostate cancer: a randomized controlled trial. JAMA 2004;292:821-7.

10. Laverdiere J, Gomez JL, Cusan L, et al. Beneficial effect of combination hormonal therapy administered prior and following external beam radiation therapy in localized prostate cancer. Int J Radiat Oncol Biol Phys 1997;37:247-52.

11. Jones CU, Hunt D, McGowan DG, et al. Radiotherapy and short-term androgen deprivation for localized prostate cancer. N Engl J Med 2011;365:107-18.

12. Mohler J, Bahnson RR, Boston B, et al. NCCN clinical practice guidelines in oncology: prostate cancer. J Natl Compr Canc Netw 2010;8:162-200.

13. Mohler JL, Kantoff PW, Armstrong AJ, et al. Prostate cancer, version 2.2014. J Natl Compr Canc Netw 2014;12:686-718.

14. Heidenreich A, Bastian PJ, Bellmunt J, et al. EAU guidelines on prostate cancer. Part 1: screening, diagnosis, and local treatment with curative intent-update 2013. Eur Urol 2014;65:124-37.

15. Viani GA, Stefano EJ, Afonso SL. Higher-than-conventional radiation doses in localized prostate cancer treatment: a meta-analysis of randomized, controlled trials. Int J Radiat Oncol Biol Phys 2009;74:1405-18.

16. Zietman AL, DeSilvio ML, Slater JD, et al. Comparison of conventional-dose vs high-dose conformal radiation therapy in clinically localized adenocarcinoma of the prostate: a randomized controlled trial. JAMA 2005;294:1233-9.

17. Peeters ST, Heemsbergen WD, Koper PC, et al. Dose-response in radiotherapy for localized prostate cancer: results of the Dutch multicenter randomized phase III trial comparing $68 \mathrm{~Gy}$ of radiotherapy with 78 Gy. J Clin Oncol 2006;24:1990-6.

18. American Joint Committee on Cancer. AJCC cancer staging manual. 7th ed. New York, NY: Springer; 2010.

19. Lukka H, Warde P, Pickles $T$, et al. Controversies in prostate cancer radiotherapy: consensus development. Can J Urol 2001;8:1314-22.

20. Nguyen $\mathrm{PL}$, Chen $\mathrm{MH}$, Beard CJ, et al. Radiation with or without 6 months of androgen suppression therapy in intermediate- and high-risk clinically localized prostate cancer: a postrandomization analysis by risk group. Int J Radiat Oncol Biol Phys 2010;77:1046-52.

21. Dubray BM, Beckendorf $V$, Guerif $S$, et al. Does short-term androgen depletion add to high-dose radiotherapy (80 Gy) in localized intermediate-risk prostate cancer: intermediary analysis of GETUG 14 randomized trial (EU-20503/ NCT00104741) [abstract]. J Clin Oncol 2011;29(Suppl):4521.

22. Alicikus ZA, Yamada Y, Zhang Z, et al. Ten-year outcomes of high-dose, intensity-modulated radiotherapy for localized prostate cancer. Cancer 2011;117:1429-37.

23. Armstrong JG, Gillham CM, Dunne MT, et al. A randomized trial (Irish clinical oncology research group 97-01) comparing short versus protracted neoadjuvant hormonal therapy before radiotherapy for localized prostate cancer. Int J Radiat Oncol Biol Phys 2011;81:35-45.

24. Crook J, Ludgate $C$, Malone $S$, et al. Final report of multicenter Canadian Phase III randomized trial of 3 versus 8 months of neoadjuvant androgen deprivation therapy before conventional-dose radiotherapy for clinically localized prostate cancer. Int J Radiat Oncol Biol Phys 2009;73:327-33.

25. Alexander A, Crook J, Jones $S$, et al. Is biochemical response more important than duration of neoadjuvant hormone therapy before radiotherapy for clinically localized prostate cancer? An analysis of the 3-versus 8-month randomized trial. Int J Radiat Oncol Biol Phys 2010;76:23-30.

26. Zapatero A, Valcarcel F, Calvo FA, et al. Risk-adapted androgen deprivation and escalated three-dimensional conformal radiotherapy for prostate cancer: does radiation dose influence outcome of patients treated with adjuvant androgen deprivation? A GICOR study. J Clin Oncol 2005;23:6561-8.

27. Cahlon 0, Zelefsky MJ, Shippy A, et al. Ultra-high dose (86.4 Gy) IMRT for localized prostate cancer: toxicity and biochemical outcomes. Int J Radiat Oncol Biol Phys 2008;71:330-7. 